\title{
The effect of intra-articular allogenic platelet rich plasma in Dunkin-Hartley guinea pig model of knee osteoarthritis
}

\author{
Himanshu Kanwat \\ Dhillon Mandeep Singh \\ Chouhan Devendra Kumar \\ Bhatia Alka \\ Saikia Biman \\ Hooda Aman
}

Postgraduate Institute of medical education and research, Chandigarh, India

\section{Corresponding Author}

Chouhan Devendra Kumar

Orthopaedics,

Postgraduate Institute of medical education

and research

Sector 12, Chandigarh, India

E-mail:drdevnim@yahoo.com

\section{Summary}

Objectives: To investigate the pathway for disease modifying effect of the PRP in osteoarthritis of knee.

Design: Two experimental models (group I and II) of Twelve Dunkin-Hartley guinea pigs each were enrolled as a part of a prospective controlled experimental study. One knee was enrolled for intervention and the other knee of the same animal used as control, the intervention being three intra-articular allogenic PRP injections given at a weekly interval. Equal volume of isotonic saline injection were given simultaneously in the control knees. Six animals from each model (subgroup IA, IIA) were euthanized at three months and the remaining six (subgroup IB, IIB) at six months post intervention. Samples of synovial fluid were collected from each knee joint for COMP level analysis by ELISA and bilateral knee joints were harvested for histopathological assessment of articular cartilage and synovium at the time of euthanasia.

Results: Mean synovial fluid COMP concentration was significantly lower in PRP treated knees $(p<0.05)$ at three months. On histological examination mean synovitis scores and synovial vascularity were significantly lower in PRP treated knees as compared to controls at both three and six months $(p<0.05)$. Additionally mean articular cartilage degeneration was significantly lower in PRP treated knees in group 1 only $(p<0.05)$.

Conclusion: Our preliminary data from the study has shown some evidence of positive influence of PRP in knee OA, possibly due to its anti-inflammatory effect and disease modifying effect, shown by short-term chondro-protective effect in PRP injected knees.

Level of evidence: V.

KEY WORDS: osteoarthritis, synovitis, COMP, biomarker, platelet rich plasma, allogenic, animal, in vivo.

\section{Introduction}

Osteoarthritis (OA) is a chronic arthropathy characterized by debilitating pain and consequent hampering of day-to-day activity, most commonly affecting the knee and the hip joints ${ }^{21}$. OA affects approximately 250 million people worldwide leading to loss in the quality of human life and significantly affects the economy ${ }^{18}$. Treatment options are limited and most of them provide only short term symptomatic relief ${ }^{15}$. Treatment modalities directed to modify the course of disease are lacking adequate evidence for widespread usage and the final outcome is often resorting to invasive procedures like joint replacement surgery for alleviation of pain ${ }^{15}$.

Platelet rich plasma (PRP) has been introduced in an attempt to modify the disease process and aid in healing of the ailing joint and symptomatic relief $8,30,28,22$. Many pre-clinical and clinical studies have been undertaken to investigate its role as a therapeutic agent for osteoarthritis and most of them have produced promising results $6,7,25,26,24$. However the mechanisms of action, long term effects and the disease modifying potential of this novel therapy are poorly understood; this needs to investigated in more detail before PRP can be considered as a "wonder drug".

In vivo studies demonstrating disease modifying effect of PRP are scarce. Kazemi et al. ${ }^{12}$ and Liu et al. ${ }^{19}$ have shown chondroprotective and anti-inflammatory effects of PRP on articular cartilage and synovium in in vivo canine and rabbit models of surgically induced OA. However, no studies have been done on a spontaneously occurring OA model such as the Dunkin Hartley guinea pigs wherein the progression and histological characteristics are comparable to human OA. No work has evaluated effects of PRP on 
both cartilage and synovial tissue, keeping in mind that $\mathrm{OA}$ is now considered a disease of the whole knee rather than being cartilage degeneration alone 21 .

\section{Methodology}

This study was a blinded controlled experimental study performed on male Dunkin-Hartley guinea pigs, proven to be a model in which knee OA occurs spontaneously and is bilaterally symmetrical, induced by increasing weight and age $^{3}$.

\section{Selection of study subjects}

After clearance from the institute animal ethics committee, 24 male Dunkin-Hartley guinea pigs weighing $600-700$ grams were obtained to be a part of the study. Two experimental models of 12 animals each were created (group I and II). One knee of each animal was selected for intervention (I) and the second knee of the same animal served as a control $(C)$ thus making a total of 24 intervention and 24 control knees. Out of each model (group I and II), 6 animals were euthanized at 3 months post intervention (subgroup IA and IIA - early outcome analysis) and the other 6 at 6 months (subgroup IB and IIB- late outcome analysis) (Flowchart 1). A separate group of 6 donor animals was taken for harvesting blood for allogenic PRP.

PRP was administered to the intervention knees once a week three times, with simultaneous administration of isotonic saline to contralateral control knees in group I. To ensure reproducibility of results and to minimize chance factor influencing the results, similar interventions were done in group II after an interval of 1 month.

\section{Preparation of platelet rich plasma}

$15 \mathrm{ml}$ of blood was drawn from one donor animal under all aseptic precautions via cardiac puncture under deep terminal anaesthesia ${ }^{23}$. Blood was collected in vials containing acid citrate dextrose (ACD) $(0.48 \%$ $\mathrm{w} / \mathrm{v}$ citric acid, $1.32 \% \mathrm{w} / \mathrm{v}$ sodium citrate and $1.47 \%$ dextrose). $1.5 \mathrm{ml}$ of this anticoagulant was used for $15 \mathrm{ml}$ of blood ${ }^{17}$. Drawn blood was analyzed for platelet count. The centrifugation protocol ${ }^{5}$ used was 20 minutes of soft spin at $800 \mathrm{rpm}$ which separated the blood into a red cell layer and a buffy coat. The buffy coat was pipetted out and subjected to hard spin for 15 minute at $2200 \mathrm{rpm}$. This yielded platelet poor plasma overlying platelet pellet at the bottom of the centrifugation tube. Two-third of the platelet poor plasma was removed and rest was gently dissolved with platelet pellet to produce a homogenous solution. $15 \mathrm{ml}$ whole blood produced $4 \mathrm{ml}$ of allogenic PRP. The obtained PRP was subjected to platelet count for quality control.

\section{Instilling PRP in guinea pig knees}

During the procedure the guinea pig was anesthetized with a mixture of Xylazine $(5 \mathrm{mg} / \mathrm{kg})+$ ketamine $(50 \mathrm{mg} / \mathrm{kg})+$ acepromazine $(1 \mathrm{mg} / \mathrm{kg})$ given at a dose of $0.1 \mathrm{ml} / \mathrm{kg}$ intramuscular injection. Under all aseptic precautions, activated PRP (1 part $0.025 \mathrm{M}$ $\mathrm{CaCl}_{2}$ to 4 parts of freshly prepared PRP) was injected in the intervention knee joint of the guinea pig through the patellar tendon with a 26 gauge needle and syringe (100 microliters) and same amount of isotonic saline was injected in the control knee of the same animal ${ }^{32}$.

\section{Collection of samples for analysis}

The animals were euthanized with $100 \mathrm{mg} / \mathrm{kg}$ of pentobarbital injected intra-peritoneally. Through the patellar tendon 200 microlitre isotonic saline was injected intra articularily ${ }^{32}$. The limb was subjected to 10 cycles of flexion and extension of knee joint to evenly distribute the injected fluid. With the same ap-

Flowchart 1. Flowchart depicting the distribution of study subjects in various groups and subgroups.

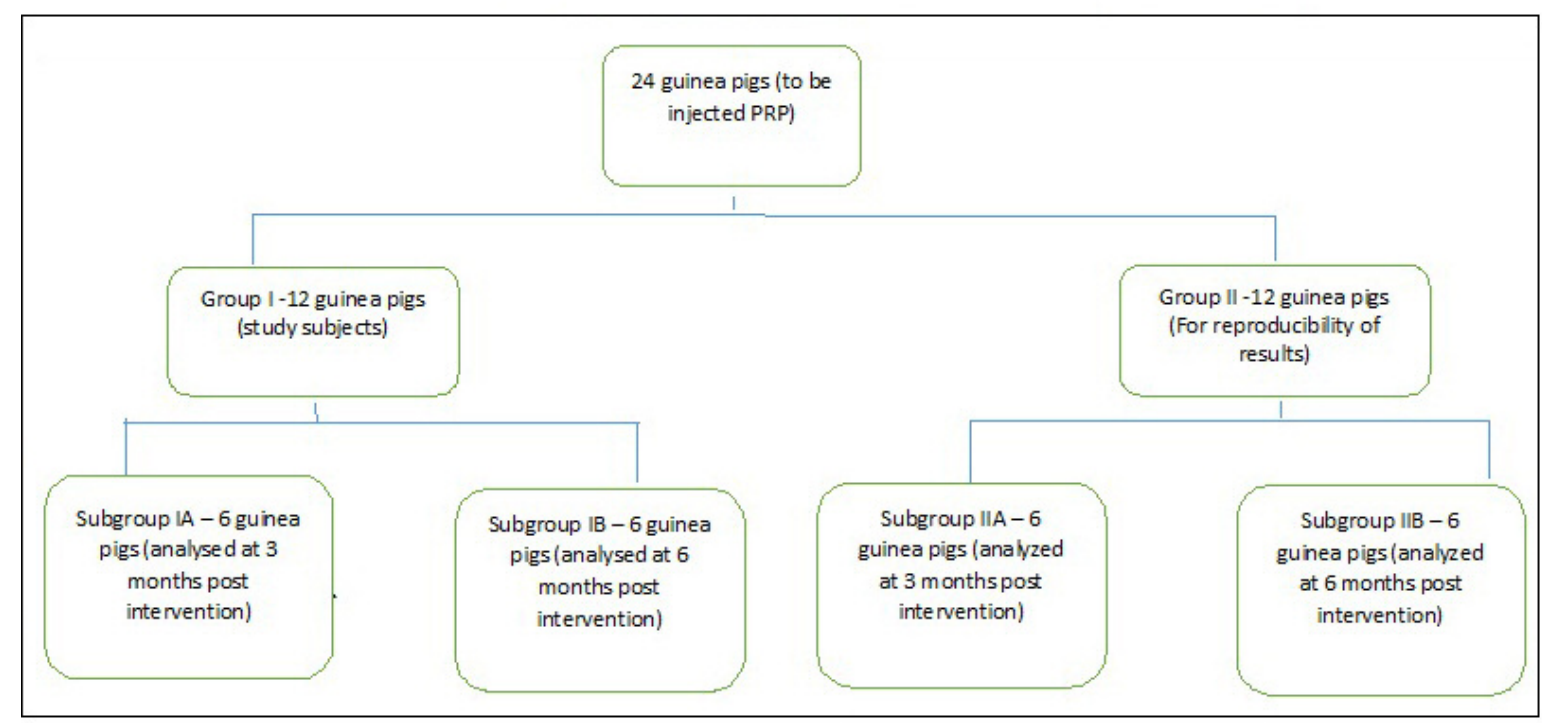


proach and a 26 gauge needle the knee was subsequently aspirated. The collected fluid was then subjected to centrifugation at $2000 \mathrm{rpm}$ for 10 minutes to remove cell debris and the supernatant collected and stored at $-80^{\circ} \mathrm{C}$ for analysis; 100 microlitre samples were thus stored. The same procedure was done on both knees.

After aspirations, the knee joints were surgically harvested. The harvested tissue was fixed in $10 \%$ formaldehyde and sent for histological processing immediately.

\section{Analysis of synovial fluid}

Synovial fluid cartilage oligomeric matrix protein (COMP) levels were measured using sandwich ELISA technique (MyBiosource Elisa kits; cat no. MBS266330).

\section{Analysis of synovium and articular cartilage his- tology}

The collected tissue samples were processed according to the algorithm outlined by the OARSI histopathology initiative ${ }^{13}$. For analysis of synovium, 4 micrometer sections were obtained and stained with hematoxylin and eosin stain. For analysis of articular cartilage, $5 \mathrm{mi}-$ crometer sections were stained with toluidine blue stain. Synovial inflammation and articular cartilage degeneration were graded according to semi-quantitative scores given and validated for use in guinea pigs by OARSI $^{13}$ (Tab. I a, b). Two independent blinded observations were made for synovial inflammation while a single blinded observation was made articular cartilage degeneration. The synovial vascularity was also assessed and $>3$ blood vessels per high power field was considered increased synovial vascularity 27.

Table I a. OARSI recommended mödified Mainkin scoríng för articulär cartilage histology ${ }^{15}$.

\begin{tabular}{|c|c|}
\hline Parameter & Description \\
\hline $\begin{array}{l}\text { Articular cartilage } \\
\text { structure }\end{array}$ & $\begin{array}{l}\text { 0) Normal, smooth, uninterrupted surface } \\
\text { 1) Mild surface irregularities (undulations) } \\
\text { 2) Irregular surface, 1-3 superficial clefts (fissures) } \\
\text { 3) }>3 \text { fissures and/or loss of cartilage in the superficial zone } \\
\text { 4) } 1-3 \text { fissures extending into the middle zone } \\
\text { 5) }>3 \text { fissures and/or loss of cartilage extending into the middle zone } \\
\text { 6) } 1-3 \text { fissures extending into the deep zone } \\
\text { 7) }>3 \text { fissures extending into the deep zone and/or loss of cartilage to deep zone } \\
\text { 8) Fissures or loss of cartilage extending to the zone of calcified cartilage }\end{array}$ \\
\hline $\begin{array}{l}\text { Proteoglycan } \\
\text { content }\end{array}$ & $\begin{array}{l}\text { 0) Uniform throughout articular cartilage } \\
\text { 1) Decreased in superficial zone only and for <half the length of the condyle or plateau } \\
\text { 2) Decreased in superficial zone for half the length or greater of the condyle or plateau } \\
\text { 3) Decreased in superficial and middle zones for <half the length of the condyle or plateau } \\
\text { 4) Decreased in superficial and middle zones for half the length or greater of the condyle or } \\
\text { plateau } \\
\text { 5) Decreased in all } 3 \text { zones for <half the length of the condyle or plateau } \\
\text { 6) Decreased in all } 3 \text { zones for half the length or greater of the condyle or plateau }\end{array}$ \\
\hline Cellularity & $\begin{array}{l}\text { 0) Normal (1/2 cells/lacuna) } \\
\text { 1) Diffuse/slight hypercellularity } \\
\text { 2) Regions of hypercellularity and clustering } \\
\text { 3) Diffuse hypocellularity }\end{array}$ \\
\hline Tidemark integrity & $\begin{array}{l}\text { 0) Intact/single tidemark } \\
\text { 1) Crossed by vessels/duplication of tidemark }\end{array}$ \\
\hline Osteophyte & $\begin{array}{l}\text { 0) No osteophyte present } \\
\text { 1) Small osteophyte } \\
\text { 2) Medium-sized osteophyte } \\
\text { 3) Large osteophyte }\end{array}$ \\
\hline
\end{tabular}


Table I b. OARSI recommended synovitis score given by Pelletier et al ${ }^{15}$.

\begin{tabular}{l|l}
\hline Parameter & Description \\
\hline Synovial Hyperplasia & 0) $1-2$ layer of cells \\
& 1) $3-5$ layers of cells \\
& 2) $>6$ layers of cells \\
\hline Villous Hyperplasia & 0) Not present \\
& 1) Few, scattered, short \\
& 2) Marked (finger like projections) \\
\hline $\begin{array}{l}\text { Degree of cellular infiltration by } \\
\text { perivascular lymphocytes and } \\
\text { mononuclear cells }\end{array}$ & 3) Marked and diffuse \\
\hline
\end{tabular}

Table II. Mean weight gain in various subgroups.

\begin{tabular}{lccc}
\hline & $\begin{array}{c}\text { Mean initial weight } \\
\text { (in grams) }\end{array}$ & $\begin{array}{c}\text { Mean weight at sacrifice } \\
\text { (in grams) }\end{array}$ & $\begin{array}{c}\text { Mean weight gain } \\
\text { (in grams) }\end{array}$ \\
\hline Subgroup IA & 723.33 & 979.69 & $252.33 \pm 71.92$ \\
\hline Subgroup IB & 716.67 & 1241 & $524.33 \pm 47.73$ \\
\hline Subgroup IIA & 668.83 & 928.67 & $214.00 \pm 14.18$ \\
\hline Subgroup IIB & 714.67 & 1212.33 & $543.50 \pm 31.97$ \\
\hline $\begin{array}{l}\text { Comments on } \\
\text { comparison }\end{array}$ & $\begin{array}{l}\text { No statistically significant difference found in weight gains between subgroups IA and IIA } \\
(\mathrm{p}=0.229) \text { and subgroups IB and IIB ( } \mathrm{p}=0.433) .\end{array}$ \\
\hline & Thus, groups I and II were comparable in terms of weight gain. \\
\hline
\end{tabular}

\section{Statistics}

The collected data was subjected to statistical analysis using SPSS v20 software. The descriptive statistics for all 4 subgroups (e.g. mean, standard deviation) were calculated for all parameters (i.e. weight, platelet counts in whole blood and PRP, synovitis scores, COMP concentration and articular cartilage scores). Weight gain and platelet counts were compared between the groups using the t-test. The synovitis scores and articular cartilage scores of the intervention and control samples were compared using t-test for significance. The results of group I and group II were compared using t-test for reproducibility between the 2 groups. Agreement between the 2 observations for synovial inflammation was calculated by reliability tests using Kronbach's alpha parameter. A p-value of $<0.05$ was considered significant.

\section{Results}

There was no statistically significant difference in the weight gain of the study subjects in group I and group II as well as between subgroups IA, IIA and IB, IIB (Tab. II). The mean platelet count in whole blood used for PRP preparation was $565,000 / \mu$ l for group I and $612,000 / \mu$ for group II (mean of 3 injections). The mean platelet count in injected PRP was

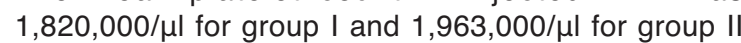
( 3 times the baseline counts) (Fig. 1).

The mean synovial fluid COMP concentration in the intervention and control samples in each subgroup was calculated (Fig. 2). The mean synovial fluid COMP concentration was higher in control samples as compared to intervention samples in each subgroup. However, the difference was statistically significant only in subgroup IA $(p=0.018)$ and trending 


\section{Platelet count in source blood}

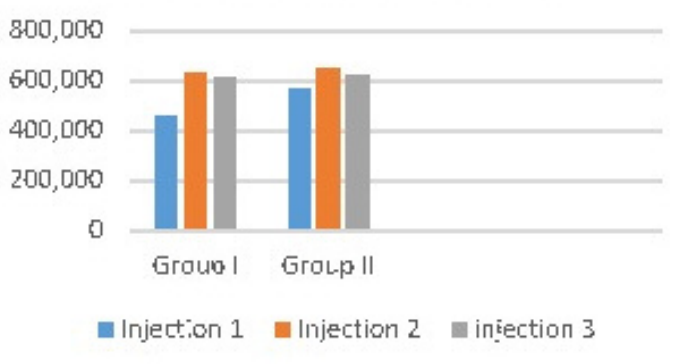

\section{Platelet count in PRP}

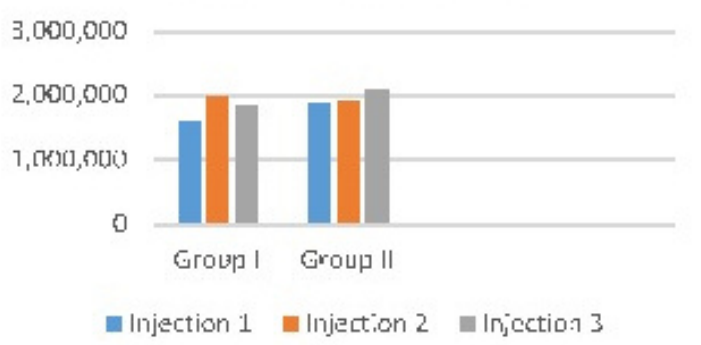

Figure 1. Mean platelet in the source blood and final PRP preparation.

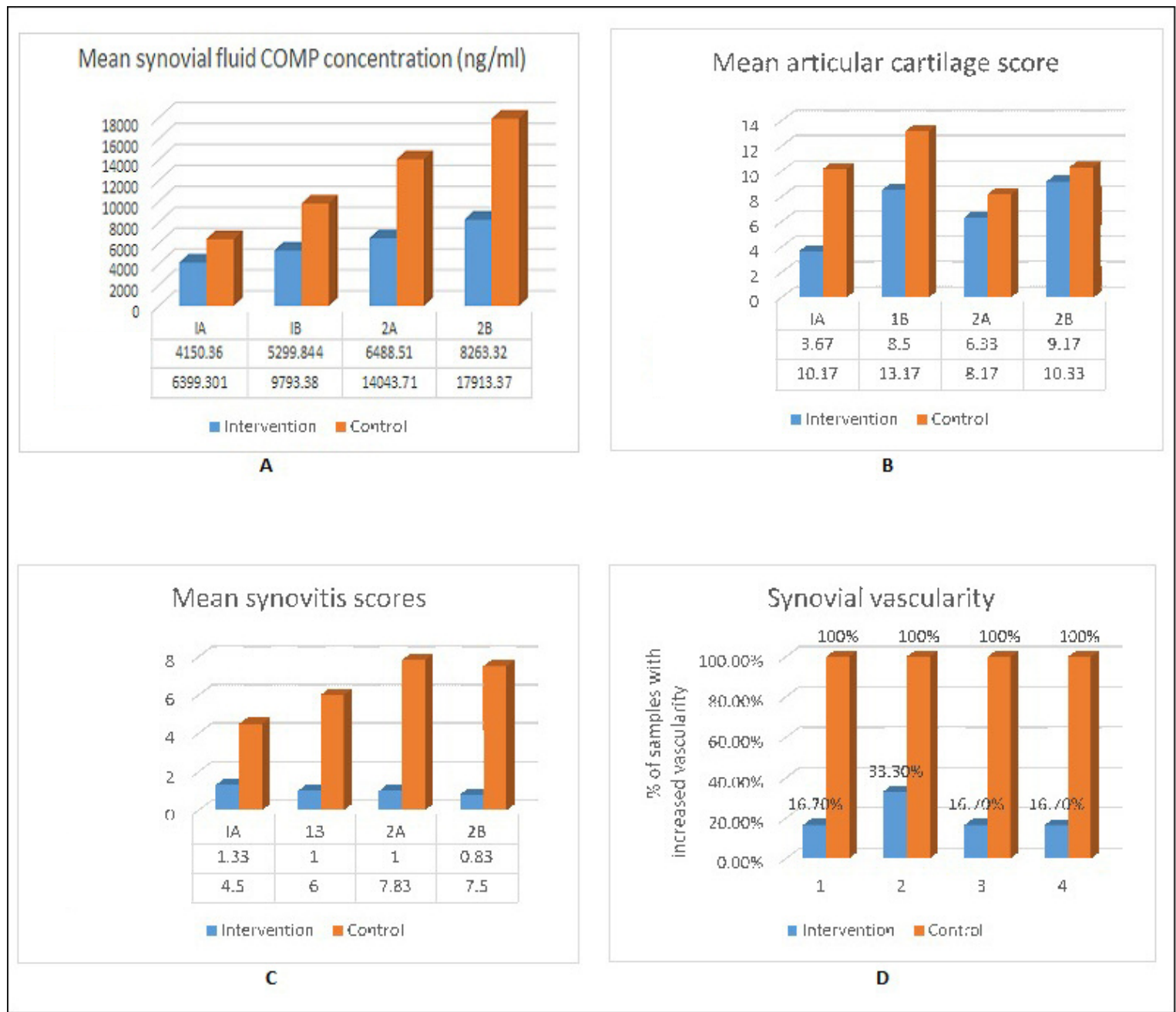

Figure 2. A) Mean synovial fluid COMP concentration [Standard deviation- intervention group [ $1 \mathrm{~A}=1733.45,1 \mathrm{~B}=5299.84$, $2 A=3606.04,28=8047.74)$, Control group $[1 A=2377.96,1 B=5985.20,2 A=8401.64,28=1.40)]$. $B$ ) Mean articular cartilage scores [Standard deviation- intervention group $[1 \mathrm{~A}=1.366,1 \mathrm{~B}=1.64,2 \mathrm{~A}=2.160,28=2.927$ ), Control group [1 $\mathrm{A}=3.488$, $18=1.472,2 A=2.401,28=4.546)$ ]. C) Mean synovitis scores [Standard deviation- intervention group [ $1 A=1.03,18=0.894$, $2 A=0.632,28=0.753)$, Control group $[1 A=3.2,1 B=2.683,2 A=0.753,28=2.258)]$. D) Graph depicting percentage of samples with increased vascularity in each group. 
towards significance in subgroup IIA $(p=0.087)$. There was no significant difference in these results between group I and II ( $p=0.06$ for control group and 0.217 for intervention group).

The mean articular cartilage scores in the intervention and control samples were calculated in each subgroup (Fig. 2). The mean articular cartilage scores were higher in control samples as compared to intervention samples in each subgroup. However, the difference was statistically significant in subgroups IA $(p=0.002)$ and subgroup IB $(p=0.0001)$. No statistically significant difference was found in subgroups IIA $(p=0.195)$ and IIB $(p=0.609)$. The improvement in articular cartilage scores observed in group I was not seen in group II. Thus, the results of group I were not reproducible in group II.

Mean synovitis scores were calculated in intervention and control samples of each subgroup (Fig. 2) for both observations. The mean synovitis scores were higher in control samples as compared to intervention samples in each subgroup. The difference was statistically significant in all subgroups except subgroup IA in first observation ( $p$-values: $I A=0.147, I B=0.001$, $\mathrm{II} A=0.0001, \mathrm{II}=0.0001)$ and in all subgroups in second observation ( $p$-values: $\mathrm{IA}=0.05, \mathrm{IB}=0.002, \mathrm{II} A=$ $0.0001, \mathrm{IIB}=0.0001)$. The results of group I were statistically comparable to that group II in both the observations. There was high agreement between the 2 observations made for synovial histology (Kronbach alpha $=0.968$ and $p$-value for agreement $=0.001)$ thus, reducing the observer bias.

Vascularity of the synovium was also analyzed (Fig. 2). Increased vascularity was found in $100 \%$ of the control samples in all subgroups whereas only $16.7 \%$ of the intervention samples showed increased vascularity in subgroups IA, IIA, IIB and $33.7 \%$ in subgroup IB. There was significantly higher vascularity in the control samples as compared to the intervention samples in each subgroup ( $p$-value: $I A=0.003, I B=0.014$, II $A=0.003$, IIB $=0.003$ ).

The results of our study show a significant improvement in synovitis and a reduction in synovial vascularity in PRP treated knees. The synovial fluid COMP levels were also significantly lower in PRP treated knees, albeit, at short term analysis only. The effect on articular cartilage, however, remains equivocal since the beneficial effect of PRP was observed into some extent in group I but was not reproducible in group II. Since the animals were of similar age and weight at the start of the study and the weight gain is comparable between groups it can be inferred that all animals had similar lesions to begin with and a similar progression ${ }^{3}$.

\section{Discussion}

Intra-articular injection of platelet rich plasma was introduced with a potential to act as a disease modifying therapy in early stages of $\mathrm{OA}^{24}$ and prevent disease progression. Many clinical studies have demon- strated alleviation of symptoms of knee OA after intra-articular PRP administration $6,7,25,26,24$. However, objective evidence of the disease modifying effect with PRP reflected as radiological or histological healing of cartilage defects, is lacking. This study was designed to investigate the biologic basis of the proposed clinical effects of intra-articular administration of PRP in an experimentally controlled scenario. The effect of PRP on articular cartilage and synovium was examined histologically along with analysis of a soluble marker of OA i.e. synovial fluid COMP levels to establish the objective parameter for evaluation of disease modification.

In vitro and in vivo studies have shown cartilage regeneration with PRP treatment. Sundman et al. ${ }^{30} \mathrm{co}-$ cultured human cartilage, harvested from knee arthroplasty samples, with PRP and found that there was increased production of endogenous hyaluronic acid and aggrecan and decreased expression of catabolic enzyme MMP-3. Saito et al. ${ }^{28}$ co-cultured adult rabbit chondrocytes in 3\% PRP and showed increased in vitro chondrocyte GAG production. Liu et al. ${ }^{19}$ demonstrated improved cartilage and subchondral bone restoration via micro CT in a rabbit model after creating $5 \mathrm{~mm}$ defects in femoral condyles and treatment with 3 weekly PRP injections as compared to controls and hyaluronic acid treated groups. Kazemi et al. ${ }^{12}$ showed healing of surgically made chondral defects in PRP treated knees in a canine model. In our study, we were able to demonstrate the histologically appreciable chondro-protective effect of PRP on the articular cartilage in model-1: however, this could not be reproduced to the same level of significance in model-2. Being observer dependent and a demanding procedure, the histological method can lead to observer and observation variation ${ }^{13}$.

On the other hand, our study has demonstrated short-term significant reduction in mean COMP level in the intervention group. The clinical relevance of the synovial fluid COMP levels lies in the fact that higher concentration of synovial and serum COMP has been positively co-related with symptomatic OA $1,2,10,16$. Thus, indirectly, when we evaluate our equivocal histopathological results along with significant COMP level changes in PRP treated knees, our results could corroborate those of Kazemi et al. ${ }^{12}$ and Liu et al. ${ }^{19}$ indicating a beneficial effect of PRP on articular cartilage.

In our study the reduction in synovial COMP level was found to be significant at 3 months post-intervention only. This observation opens the question of reinjection after certain periods or the use of sustained release mechanism for maximal benefit, as also shown by Saito et al. ${ }^{28}$. They demonstrated increased expression of glycoprotein core mRNA in cartilage treated with PRP impregnated gelatin microspheres (PRP-M) administered twice in a surgically induced (via ACL transection) rabbit OA model. Histologically, the cartilage showed less degradation in the PRP-M treated group. However, mRNA expression and cartilage degeneration was not significantly improved in 
knees treated with PRP only.

It is important to note that in comparison to our study, other studies have limitations of either being in vitro studies, which lack the mechanical environment, or in vivo studies with surgically created chondral defect induced OA, which lacks the biological milieu of degenerative OA found in humans 9,14 .

Synovitis is considered as one of the important local biological factors for symptomatic $\mathrm{OA}^{4,29}$. In our study with two different histological observations, we could note the significant reduction in the synovitis in intervention knees compared to control knees. Increased vascularity in the synovium is a harbinger of chronic synovitis due to inflow of inflammatory cells and the consequent accumulation of leukotrienes and other affecters of inflammation, leading to pain and degradation of the cartilage ${ }^{31}$. Our findings corroborate the findings of Liu et al. ${ }^{20}$, as our results are in concert with theirs. This may give a histologic basis for reduction of pain and improvement in quality of life after intra-articular PRP injections, as observed in various clinical and experimental studies $6,7,25,26,24$. The antiinflammatory effect of the PRP was demonstrable at both 3 months and 6 months post PRP injection. The improvement in synovitis in PRP treated knees was reproducible in both the groups (group I and II). Additionally, synovial vascularity was found to be significantly lower in intervention samples as compared to the control samples in all four subgroups ( $p$-value $<0.05)$.

Being an experimental study, extrapolation of our results in the clinical scenario has some limitations; nevertheless our study has certain strong points which makes the data from our study of some clinical relevance.

First is the selection of animal for experimental study; Dunkin-Hartley guinea pigs were chosen as a model because of certain peculiarities like spontaneously occurring $O A$, which reduces cost and interventions $^{3,13}$. The spontaneous lesions are well co-related with weight gain and age of the animal such that specific lesions can be found in guinea pigs of a certain age and weight ${ }^{3}$. The progression and appearance of joint pathology in these guinea pigs is influenced similarly by the well-known risk factors of human disease such as age, weight gain, sedentary lifestyle and mechanical loading ${ }^{13}$. The pathology initially appears in the medial compartment of knee, with femoral lesions lagging behind tibial lesions, which conforms to the pattern seen in idiopathic, non-traumatic OA knee in humans ${ }^{13}$. In addition to the histologic similarity, various soluble OA markers found in humans are detectable and validated in the guinea pig model ${ }^{11}$.

Secondly, since the disease is bilaterally symmetrical in this particular experimental model, we were able to use the contralateral knee, with similar changes, as a control for the knee in which PRP was administered. Thirdly, we have utilized allogeneic PRP prepared from guinea pig blood. Allogeneic PRP has been shown to be non-immunogenic in local application and ensures uniform quantity and quality of platelets $^{33}$. Thus, inter- and intra-organism variability associated with autologous PRP was eliminated by using single source for PRP (allogenic), which permits a more consistent preparation.

Fourthly, being an in vivo study the anabolic effect on the cartilage can be evaluated in an environment that allows mechanical loading and is nearest to the clinical scenario ${ }^{9}$. It is important to note that experimentally induced chondral defects lack the intra-articular milieu like degenerative $O A$, which is not merely failure of cartilage alone ${ }^{21}$. However our model of spontaneous OA reproduces the milieu of naturally occurring disease ${ }^{14}$. The temporal progression mimics human knee OA, and is well controlled, unlike experimentally induced OA which may progress rapidly ${ }^{13,14}$. Fifthly, analysis of a biomarker for evaluating the cartilage metabolism supports and substantiates the histological findings. Study of biomarkers being observer independent, results are more dependable when combined with histology.

Sixthly, the study of the effect at different time intervals after intervention allows the analysis of the weaning off effect with the passage of time. Future research, directed to look at the frequency of the injection to obtain the persistent control over progression of $\mathrm{OA}$, is thus imperative.

\section{Conclusion}

Our study showed that intra-articular PRP injection results in reduction of synovial inflammation and vascularity as compared to controls, which may be the biological basis of improvement in pain after PRP injection, in addition to short term chondro-protective effect. The anti-inflammatory effect was present at both 3 months and 6 months post-intervention, which can correspond to short term and long term effect in humans respectively, keeping in view the short lifespan of the guinea pigs and the fact that knee OA in guinea pigs is temporally proportionate to that found in human beings. However, the effect on articular cartilage remains somewhat equivocal, however, since the significant results of histological analysis of the cartilage were not reproducible in all subjects in our limited study. Nevertheless the evaluation of COMP levels, which is a marker of cartilage metabolism, demonstrated that the chondro-protective effect of PRP is present at short term analysis but it may not be present over a prolonged duration. This opens up avenues for future research regarding the timing of PRP injections to allow its effect over prolonged durations.

\section{Acknowledgement}

The funding was provided by PGIMER, Chandigarh via research grant. No external funding recruited. 


\section{Conflict of interest}

The Authors declare no conflict of interest.

\section{Author contribution statement}

The primary Author was involved in the concept, design of study, performing the experiments, data collection and analysis. Dr. Devendra Chouhan gave the concept behind the study, the study design and edited the manuscript. Dr. Dhillon was the senior guide and involved in study design, interpreting the result and editing the manuscript. Dr. Alka was involved in histological analysis and PRP preparation and $\mathrm{Dr}$. Biman was involved in performing the experiment and interpreting biomarker results. Dr. Aman was involved in writing the manuscript and data collection.

\section{References}

1. Bauer DC, Hunter DJ, Abramson SP, Attur M, Corr M, Felson D, et al. Classification of osteoarthritis biomarkers: a proposed approach. Osteoarthritis Cartil. 2006;14:723-7.

2. Bellucci F, Meini S, Cucchi P. Synovial fluid levels of bradykinin correlate with biochemical markers for cartilage degradation and inflammation in knee osteoarthritis. Osteoarth Cartil . 2013;21:1774-1788.

3. Bendele AM. Animal models of osteoarthritis. J Musculoskel Neuron Interact 2001;1:363-376.

4. Brokaar BJE, Facsinay AL, Yusuf E. Degree of synovitis on MRI by comprehensive whole knee semi-quantitative scoring method correlates with histologic and macroscopic features of synovial tissue inflammation in knee osteoarthritis. Osteoarth Cartil. 2014;22:1606-1613 .

5. Dohan DM, Rasmusson L, Albrektsson T. Classification of platelet concentrates: from pure platelet rich plasma (P-PRP) to leukocyte and platelet rich fibrin (L-PRF). Trends Biotechnol. 2009; 27:158-167.

6. Duymus T, Mutlu S, Dernek B, Komur B, Aydogmus S, Kesiktas F. Choice of intra-articular injection in treatment of knee osteoarthritis: Platelet-rich plasma, hyaluronic acid or ozone options. Knee Surg Sports Traumatol Arthrosc. 2016. Epub ahead of print.

7. Filardo G, Di Matteo B, Di Martino A, Merli M, Cenacchi A, Fornasari $P$ et al. Platelet-rich plasma intraarticular knee injections show no superiority versus viscosupplementation: a randomized controlled trial. Am J Sports Med. 2015;43:15751582.

8. Fortier LA, Barker JU, Strauss JE, McCarell TM, Cole BJ. The role of growth factors in cartilage. Clin Orthop Relat Res. 2011; 469:2706-2715.

9. Griffin T M, Farshid. The role of mechanical loading in the onset and progression of osteoarthritis. Exere Sport Sci Rev. 2005;33:195-200.

10. Hunter DJ, Li J, LaValley M, Bauer D, Nevitt M, DeGroot J et al. Cartilage markers and their association with cartilage loss on magnetic resonance imaging in knee osteoarthritis: The Boston knee study. Arthritis Res Ther. 2007; 9:R108.

11. Huebner JL, Kraus MS, Kraus VB. Assessment of utility of biomarkers of osteoarthritis in guinea pig. Osteoarth Cartil. 2006;14:923-930.

12. Kazemi D, Fakhrion A. Luekocyte and platelet rich plasma (LPRP) versus leukocyte and platelet rich fibrin (L-PRF) for artic- ular cartilage repair of the knee: A comparative evaluation in an animal model. Iran Red Crescent Med J. 2015;17:1945-49.

13. Kraus VB, Huebnery JL, DeGrootz J. The OARSI histopathology initiative recommendations for histological assessments of osteoarthritis in guinea pig. Osteoarth Cartil. 2010:S35-S52.

14. Kuyinu E, Narayanan G, Nair L, Laurencin C. Animal models of osteoarthritis: classification, update and measurement of outcomes. J Othop Surg Res. 2016;6:1192-1196.

15. Langworthy MJ, Saad A, Langworthy NM. Conservative treatment modalities and outcomes for osteoarthritis: the concomitant pyramid of treatment. Phys Sportsmed. 2010;38:133-45

16. Larsson E, Erlandsson HH, Larsson A. Corticosteroid treatment of experimental arthritis retards cartilage destruction as determined by histology and serum COMP. Rheumatology. 2004;43:428-34.

17. Lei $H$, Gui L, Xiao R. The effects of anticoagulants on the quality and biological efficacy of platelet rich plasma. Clin Biochem. 2009;42:1452-1460.

18. Lim SS, Vos T, Flaxman AD, Danaei G, Shibuya K, Rohani H, et al. A comparative risk assessment of burden of disease and injury attributable to 67 risk factors and risk factor clusters in 21 regions, 1990-2010: a systematic analysis for the Global Burden of Disease Study. 2010. Lancet. 2012;380:2224-60

19. Liu J, Song W, Yuan T, Xu Z, Jia W, Zhang C. A comparison between platelet rich plasma and hyaluronic acid on healing of cartilage defects. Plos one. 2014;9:e97293.

20. Liu J, Yuan T, Zhang C. Effect of platelet rich plasma on synovitis of rabbit knee. Chinese journal of reparative and reconstructive surgery. 2011;25:285-90.

21. Loeser RF, Goldring SR, Scanzello CR, Goldring MB . Osteoarthritis: A disease of joint as an organ. Arthritis Rheum. 2012;64:1697-1707.

22. Mifune $Y$. The effect of platelet rich plasma on the regenerative therapy of muscle derived stem cells for articular cartilage repair. Osteoarth Cartil. 2013;21:175-185.

23. Parasuraman S, Raveendran R, Kesavan R. Blood sample collection in small laboratory animals. J Pharmacol Pharmacother. 2010;1:87-93.

24. Patel S, Dhillon MS, Aggarwal S, Marwaha N, Jain A. Treatment with platelet-rich plasma is more effective than placebo for knee osteoarthritis: A prospective, double blind, randomized trial. Am J Sports Med. 2013;41: 356-364.

25. Paterson K, Nicholls M, Bennell K, Bates D. Intraarticular injection of photo-activated platelet-rich plasma in patients with knee osteoarthritis: A double-blind, randomized controlled pilot study. BMC Musculoskelet Disord. 2016;17:67.

26. Raeissadat S, Rayegani S, Hassanabadi H, Fathi M, Ghorbani $\mathrm{E}$, Babaee M, et al. Knee osteoarthritis injection choices: Platelet-rich plasma (PRP) versus hyaluronic acid (a one-year randomized clinical trial). Clin Med Insights Arthritis Musculoskelet Disord. 2015;8:1-8.

27. Rooney M, Condell D, Quinlan W, Daly L, Whelan A, Feighery $\mathrm{C}$, et al. Analysis of the histologic variation of synovitis in rheumatoid arthritis. Arthritis Rheum. 1988;31:956-63.

28. Saito M, Takahashi KA, Arai Y, Inoue A, Sakao K, Tonomura $\mathrm{H}$ et al. Intra-articular administration of platelet rich plasma with biodegradable gelatin hydrogel microspheres prevents osteoarthritis progression in the rabbit knee. Clin Exp Rheumatol. 2009; 27:201-207.

29. Stoppiello LA, Mapp PI, Wilson D, Hill R, Scammell B, Walsh D. Structural associations of symptomatic knee osteoarthritis. Arthritis Rheum. 2014; 66:3018-3027.

30. Sundman EA, Cole BJ, Karas V, Della V, Tetriault MW, Fortier $\mathrm{LA}$, et al. The anti-inflammatory and matrix restorative mechanisms of platelet-rich plasma in osteoarthritis. Am J Sports Med. 2014;42:35-41.

31. Walsh DA, Bonnet CS, Turner EL, Wilson E, Situ M, McWilliams DF. Angiogenesis in the synovium and at the os- 
teochondral junction in osteoarthritis. Osteoarthrits cartilage. 2007;15:743-51.

32. Wei L, Fleming BC, Sun X, Teeple E, Wu W, Jay G, et al. A comparison of differential biomarkers of $O A$ with and without post-traumatic injury in the Hartley guinea pig model. $\mathrm{J}$ orthop
Res. 2010;28:900-906.

33. Zhang ZY, Huang AW, Fan JJ, Wei K, Jin D, Chen B, et al. The potential use of allogeneic platelet rich plasma for large bone defect treatment: immunogenicity and defect healing efficacy. Cell transplant. 2013;22:175-87. 